XX Міжнародний симпозіум «Методи дискретних особливостей в задачах математичної фізики/Discrete Singularities Methods in Mathematical Physics»,

УДК 517.968 МДОЗМФ/DSMMPh-2021

MSC 45E05

\title{
Regularization of some perturbed integral operators in the spaces $L_{p}$
}

\author{
Vasile Neagu \\ Moldova State Universyty, Chișinău \\ E-mail: vasileneagu45@gmail.com
}

The article presents some generalizations and refinements of the article [1]: examples of integral (noncompact) operators with point wise singularities which are admissible perturbations of the Noetherian operators are constructed; a connection between the regularizes of the perturbed and original operators is established and the equality between the indices of the perturbed and the original operators is proved. The presented results are based on the formulas obtained in this paper for the composition of an operator with the Cauchy kernel and the operators with point wise singularities.

Key words: perturbed singular operators, noetherian conditions.

\section{Регуляризація деяких збурених інтегральних операторів у просторах $L_{p}$

\author{
Василе Нягу \\ Молдавський державний університет, Кичинів \\ E-mail:vasileneagu45@gmail.com
}

У статті представлені деякі узагальнення і уточнення статті [1]: будуються приклади інтегральних (некомпактної) операторів 3 точковими особливостями, які є допустимими збуреннями нетерових операторів; встановлюється зв'язок між регуляризатора збуреного і вихідного операторів, доводиться рівність між індексамі збуреного і вихідного операторів. Представлені результати грунтуються на отримані в даній роботі формули композиції оператора з ядром Коші і операторами 3 точковими особливостями.

Ключевые слова: збурені сингулярні оператори, нетерові умови.

\section{Introduction}

We remind that an operator $A \in L(B)$ admits regularization if there exist operators $M_{1}, M_{2} \in L(B)$ such that $A M_{1}=I+T_{1}$ (left regularization) and $M_{2} A=I+T_{2}$ (right regularization), where $T_{1}$ and $T_{2}$ are compact operators in space $B$. The class of operators admitting regularization is of particular interest, since the operators of this class have the following properties (F. Noether's theorems):

1) The equation $A x=y$ is solvable if and only if its right-hand side is orthogonal to all solutions of the equation $A^{*} \varphi=0$. This condition is equivalent to the condition that the set of values of the operator $A$ is a subspace, or the equality

$$
\operatorname{Im} A=\bigcap_{f \in \operatorname{Ker} A^{*}} \operatorname{Ker} f
$$

is true.

2) The equations $A x=0$ and $A^{*} \varphi=0$ have a finite number of linearly independent solutions.

Operators with properties 1) and 2) are called Noetherian operators and represent essential generalizations of the class of operators of the form $I+T$, where $T$ is a compact operator for which the well-known Fredholm theorems hold. If conditions 1) and 2) are

(c) Нягу B., 2021 
satisfied, then the number $\operatorname{dim} \operatorname{Ker} A-\operatorname{dim} \operatorname{Ker} A^{*}$ is called the index of a Noetherian operator $A$ and is denoted by $\operatorname{Ind} A$.

Let us denote by the $N(B)$ the set of all Noetherian operators acting in a Banach space $B$ and let $H$ be a Hilbert space. It is well known that if an operator $K \in L(H)$ and has the property $A+K \in N(H)$ for every $A \in N(H)$, then $K$ is completely continuous.

And what will be, if we require that the implication $A \in N(H)$ implies " $A+K \in N(H) \Leftrightarrow A \in N(H)$ ", but say, for all singular integral Noetherian operators. Is $K$ necessarily completely continuous in this case? It turns out that it is not necessary. Examples of such operators can be found in [1,2-4], and such examples are given in this paper.

\section{Perturbation of Noetherian singular operators}

In the monographs of N.I. Muskhelishvili and F.D. Gakhov, an operator is called complete singular integral operator if it has the form

$$
(A \varphi)(t)=a(t)+\frac{1}{\pi i} \int_{\Gamma} \frac{k(\tau, t) \varphi(\tau)}{\tau-t} d \tau,
$$

where $a(t)$ and $k(\tau, t)$ are functions satisfying the Hölder condition on $\Gamma$ and $\Gamma \times \Gamma$, respectively, and the integral is understood in the sense of the principal value. The operator $A$, defined by equality (1), can be represented in the form $A=a I+b S+T$, where $b(t)=k(t, t)$, and $T$ is the integral operator with kernel

$$
k_{0}(\tau, t)=\pi i \frac{k(\tau, t)-k(t, t)}{\tau-t} .
$$

In the case when $k(\tau, t)$ satisfies the Hölder condition on $\Gamma \times \Gamma$, the kernel (2) has a weak singularity; therefore, the operator $T$ is completely continuous in the space $L_{p}(\Gamma)$. Due to this, the operator $A$ is Noetherian in the space $L_{p}(\Gamma)$, if and only if the operator

$$
A_{0}=a I+b S
$$

is Noetheran. Operator $A_{0}$ is called the characteristic part of the operator $A$. In this connection, Noether's theory of singular operators was developed mainly for characteristic operators. Significant successes have been achieved in this direction: there are obtained criteria to be Noetherian for such operators with piecewise continuous coefficients, with coefficients having discontinuities of almost periodic type, with arbitrary coefficients from $L_{\infty}(\Gamma)$. However, in many problems of mechanics, physics and other areas that lead to singular equations, not characteristic operators appear, but complete ones. In this regard, it becomes necessary to study the complete singular operators (1) with functions and $k(\tau, t)$ not necessarily satisfying the Hölder condition. The main difficulty here is that the operator $T$ with kernel (2) may turn out to be not completely continuous (not compact) or (more importantly) ceases to be an $\Phi$-admissible perturbation 
XX Міжнародний симпозіум «Методи дискретних особливостей в задачах математичної фізики/Discrete Singularities Methods in Mathematical Physics»,

\section{МДОЗМФ/DSMМPh-2021}

Let's show this on an example. Let $\Gamma_{0}$ be the unit circle, $\chi(t)$ be the characteristic function of the $\{\operatorname{Im} t>0\} \cap \Gamma_{0} ; k(\tau, t)=\chi(t)-\chi(\tau), \lambda \in \mathbb{C}$

$$
(A \varphi)(t)=\lambda \varphi(t)+\frac{1}{\pi i} \int_{\Gamma_{0}} \frac{k(\tau, t) \varphi(\tau)}{\tau-t} d \tau .
$$

In this example, $k(t, t)=0$, therefore, the characteristic part of the operator $A$ is a scalar operator $\left(A_{0} \varphi\right)(t)=\lambda \varphi(t)$. The operator $A$ in this example can be represented in the form $A=\lambda I+\chi S-S \chi I$, whence it follows that it belongs to the algebra $A_{p}$, generated by singular integral operators with piecewise continuous coefficients. It was shown in (2) that on the algebra $A_{p}$ one can introduce the symbol $\left(\gamma_{\tau, \mu}\right)\left((\tau, \mu) \in \Gamma_{0} \times[0,1)\right)$, which on the generators of $A=\lambda I+\chi S-S \chi I$ and $a I$ takes the form

$$
\gamma_{\tau, \mu}(a I)=\left\|\begin{array}{cc}
a(t+0) f_{p}(\mu)+a(t-0)\left(1-f_{p}(\mu)\right) & (a(t+0)-a(t-0)) h_{p}(\mu) \\
(a(t+0)-a(t-0)) h_{p}(\mu) & a(t+0)\left(1-f_{p}(\mu)\right)+a(t-0) f_{p}(\mu)
\end{array}\right\|,
$$

where

$$
f_{p}(\mu)=\left\{\begin{array}{l}
\frac{\sin \theta \mu}{\sin \theta} e^{i \theta(\mu-1)}\left(\theta=\frac{\pi(p-2)}{2}\right), \text { for } p \neq 2, \\
\mu, \text { for } p=2,
\end{array}\right.
$$

and $h_{p}(\mu)$ - is some fixed continuous branch of the function $\sqrt{f_{p}(\mu)\left(1-f_{p}(\mu)\right)}$.

In particular, for the operator $A=\lambda I+\chi S-S \chi I$ with $p=2$ we have: $\operatorname{det} \gamma_{\tau, \mu}(A)=\lambda^{2}$ for $t \neq \pm 1$ and $\operatorname{det} \gamma_{\tau, \mu}(A)=\lambda^{2}+4 \mu(1-\mu)$ for $t= \pm 1$. An operator $A$ is Noetherian in $L_{2}(\Gamma)$ if and only if $\lambda^{2}+4 \mu(1-\mu) \neq 0$ for all $\mu \in[0,1]$. This is equivalent to $\lambda \neq i$, where $t \in[-1,1]$.

Thus, for $\lambda=i \tau$, where $\tau \in[-1,1] \backslash\{0\}$, the operator $A$ is not Noetherian, but its characteristic part $A_{0}$ is Noetherian. This implies that the operator $M=A-A_{0}$ is not a $\Phi$-admissible perturbation of the characteristic part of the operator $A$. This also implies that $M$ is not compact.

For this operator, we managed to obtain criteria for Noetherian property due to the fact that we embedded it in the algebra $A_{p}$ (see [5-6]). You can do the same with some other complete operators. This work will describe one class of such operators.

In what follows, we will consider the perturbation of the characteristic operators by operators of the following form

$$
(K \varphi)(t)=\sum_{k=1}^{m} c_{k}(t)\left(M_{k} \varphi\right)(t) \quad\left(c_{k} \in L_{\infty}(\Gamma)\right),
$$

where

$$
\left(M_{k} \varphi\right)(t)=\frac{1}{\pi i} \int_{\Gamma} \frac{\varphi(\tau)}{\tau-t-\alpha_{k}} d \tau \quad(t \in \Gamma)
$$


and $\alpha_{k}(\neq 0)$ are some complex numbers. First of all, note that if the function $\tau-t-\alpha_{k}$ vanishes at some point $(\tau, t) \in \Gamma \times \Gamma$, then the corresponding operator $M_{k}$ is not compact. This follows from the following theorem.

Theorem 1. Let $\Gamma_{k}=\left\{z: z=t-\alpha_{k}, t \in \Gamma\right\}$. If $\Gamma \cap \Gamma_{k}=\varnothing$, the operator $M_{k}$ is not compact in the space $L_{p}(\Gamma)$.

Proof. Suppose that the operator $M_{k}$ is compact in the space $L_{p}(\Gamma)$. Let $\gamma=\Gamma \cup \Gamma_{k}$ and $t_{0}$ be one of the intersection points of the contours $\Gamma$ with $\Gamma_{k}$. In the space $L_{p}(\Gamma)$ consider the singular operator defined by the equality

$$
A=a I+b S_{\gamma},
$$

where $a(t)$ and $b(t)$ are continuous at each point $t \in \gamma \backslash\left\{t_{0}\right\}$ and satisfy the conditions:

$a(t \pm 0) \pm b(t \pm 0) \neq 0, \quad\left(a\left(t_{0}-0\right)+b\left(t_{0}-0\right)\right) /\left(a\left(t_{0}-0\right)-b\left(t_{0}-0\right)\right)=i \quad$ and $\left(a\left(t_{0}+0\right)+b\left(t_{0}+0\right)\right) /\left(a\left(t_{0}+0\right)-b\left(t_{0}+0\right)\right)=1$. Under these conditions, the operator $A$ is not Noetherian [6] in space $L_{2}(\gamma)$. Operator $R$, acting by rule

$$
(R \varphi)(t)=\left(\varphi(t), \varphi\left(t-\alpha_{k}\right)\right), \quad t \in \Gamma,
$$

is the reversible operator from $L\left(L_{2}(\gamma), L_{2}^{2}(\Gamma)\right)$. Let $\psi \in L_{p}(\Gamma)$ and consider the equation

$$
A \varphi=a \varphi+b S_{\gamma} \varphi=\psi .
$$

This equation can be rewritten as a system of two equations: in one equation $t \in \Gamma$, and in the second equation $t \in \Gamma_{k}$,

$$
\begin{cases}a(t) \varphi(t)+\frac{b(t)}{\pi i} \int_{\Gamma} \frac{\varphi(\tau)}{\tau-t} d \tau+\frac{b(t)}{\pi i} \int_{\Gamma_{k}} \frac{\varphi(\tau)}{\tau-t} d \tau=\psi(t), \quad t \in \Gamma, \\ a(t) \varphi(t)+\frac{b(t)}{\pi i} \int_{\Gamma} \frac{\varphi(\tau)}{\tau-t} d \tau+\frac{b(t)}{\pi i} \int_{\Gamma_{k}} \frac{\varphi(\tau)}{\tau-t} d \tau=\psi(t), \quad t \in \Gamma_{k} .\end{cases}
$$

In the integral $\int_{\Gamma_{k}} \frac{\varphi(\tau)}{\tau-t} d \tau$ we change the variables $\tau \rightarrow \tau-\alpha_{k}$ and in the second equation of the resulting system, replace $t$ by $t-\alpha_{k}$. We get

$$
\left\{\begin{array}{l}
a_{1}(t) \varphi_{1}(t)+\frac{b_{1}(t)}{\pi i} \int_{\Gamma} \frac{\varphi_{1}(\tau)}{\tau-t} d \tau+\frac{b_{1}(t)}{\pi i} \int_{\Gamma} \frac{\varphi_{2}(\tau)}{\tau-t-\alpha_{k}} d \tau=\psi_{1}(t), \quad t \in \Gamma, \\
a_{2}(t) \varphi_{2}(t)+\frac{b_{2}(t)}{\pi i} \int_{\Gamma} \frac{\varphi_{2}(\tau)}{\tau-t} d \tau+\frac{b_{2}(t)}{\pi i} \int_{\Gamma} \frac{\varphi_{1}(\tau)}{\tau-t+\alpha_{k}} d \tau=\psi_{2}(t), \quad t \in \Gamma,
\end{array}\right.
$$

where the notation $f_{1}(t)=f(t), f_{2}(t)=f\left(t-\alpha_{k}\right)(t \in \Gamma)$ is used. Thus, the operator $R A R^{-1}$ has the form

where

$$
R A R^{-1}=\left\|\begin{array}{cc}
a_{1} I+b_{1} S_{\Gamma} & b_{1} M_{k} \\
b_{2} N_{k} & a_{2} I+b_{2} S_{\Gamma}
\end{array}\right\|,
$$


As

$$
\left(S_{\Gamma} \varphi\right)(t)=\frac{1}{\pi i} \int_{\Gamma} \frac{\varphi(\tau)}{\tau-t} d \tau, \quad\left(N_{k} \varphi\right)(t)=\frac{1}{\pi i} \int_{\Gamma} \frac{\varphi(\tau)}{\tau-t+\alpha_{k}} d \tau, t \in \Gamma .
$$

$$
\mu \frac{a_{j}(t-0)+b_{j}(t-0)}{a_{j}(t-0)-b_{j}(t-0)}+(1-\mu) \frac{a_{j}(t+0)+b_{j}(t+0)}{a_{j}(t+0)-b_{j}(t+0)} \neq 0 \quad(t \in \Gamma, 0 \leq \mu \leq 1, j=1,2),
$$

then the operators $a_{j} I+b_{j} S_{\Gamma}(j=1,2)$ are Noetherian in the space $L_{2}(\Gamma)$. Then equality (7) (taking into account the compactness of the operators $M_{k}$ and $N_{k}$ ) implies that the operator $A$ is Noetherian in the space $L_{2}(\Gamma)$. The resulting contradiction proves that the operator $M_{k}$ is not compact in the space $L_{2}(\Gamma)$. Since, in addition, the operator $M_{k}$ is bounded in all spaces $L_{p}(\Gamma)(1<p<\infty)$, by virtue of M. Krasnoselsky's theorem [7] $M_{k}$ is not compact in any space $L_{p}(\Gamma)$. The theorem is proved.

\section{Main result}

To present the main results, we need to introduce some notation. Let $\Gamma_{k}=\left\{\varsigma: \varsigma=t-\alpha_{k}, t \in \Gamma\right\}$ and $\tilde{\Gamma}_{k}=\left\{\varsigma: \varsigma=t+\alpha_{k}, t \in \Gamma\right\}$. If the contour $\Gamma_{k}$ has no points in common with $\Gamma$, then, obviously, the operator $M_{k}$, defined by equality (6) is completely continuous in the space $L_{p}(\Gamma)$ and does not affect the Noetherian character of singular operators of the form $A_{0}=a P+b Q+T\left(P=\frac{1}{2}(I+S), P=\frac{1}{2}(I-S)\right)$. In this connection, in what follows we will assume that the numbers $\alpha_{k}(k=1, \ldots, m)$ are such that $\Gamma \cap \Gamma_{k} \neq \varnothing$. For the sake of simplicity, we will assume that $\Gamma$ is the unit circle: $\Gamma=\{t:|t|=1\}$. We also note here that the results of the paper are valid for any closed Lyapunov contour $\Gamma$, with the property that $\Gamma$ and $\Gamma_{k}$ intersect at a finite number of points.

Let $t_{k}^{(1)}, t_{k}^{(2)}$ be the intersection points of the contours $\Gamma$ and $\Gamma_{k}(k=1, \ldots, m)$ and $t_{k}^{(3)}, t_{k}^{(4)}$ points of intersection of the contours $\Gamma$ and $\tilde{\Gamma}_{k}: t_{k}^{(3)}=t_{k}^{(1)}+\alpha_{k}$ and $t_{k}^{(4)}=t_{k}^{(2)}+\alpha_{k}$. Let $N_{k}$ denotes the set of all functions from $L_{\infty}(\Gamma)$, that are continuous in some neighborhoods $u\left(t_{k}^{(j)}\right)$ of points $t_{k}^{(j)} \quad(j=1,2,3,4)$. Let $a \in N_{k}$ and $u_{k}^{(j)}$ $(j=1,2,3,4)$ be some neighborhoods of points $t_{k}^{(j)}$, in which the function $a(t)$ is continuous. Put $\gamma_{k}=\cup_{j=1}^{4} u_{k}^{(j)}$.

Theorem 2. Let $a \in N_{k}$, then there exists a function $a_{k} \in N_{k}$ such that $a_{k}(t)=1$ for $t \in \Gamma \backslash \gamma_{k}$ and the operator $N=M_{k} a I-a_{k} M_{k}$ is completely continuous in $L_{p}(\Gamma)$.

We denote by $l_{k}^{(1)}\left(\tilde{l}_{k}^{(1)}\right)$ the part of the contour $\Gamma$, lying inside the region bounded by the contour $\Gamma_{k}=\left\{\varsigma: \varsigma=t-\alpha_{k}, t \in \Gamma\right\} \quad\left(\tilde{\Gamma}_{k}=\left\{\varsigma: \varsigma=t+\alpha_{k}, t \in \Gamma\right\}\right)$, and let $l_{k}^{(2)}=\Gamma \backslash l_{k}^{(1)}\left(\tilde{l}_{k}^{(2)}=\Gamma \backslash \tilde{l}_{k}^{(1)}\right)$.

Theorem 3. The following equalities hold 
where

$$
M_{k} S=h_{k} M_{k}, S M_{k}=M_{k} \tilde{h}_{k} I
$$

$$
h_{k}(t)=\left\{\begin{array}{ll}
1, & \text { for } t \in l_{k}^{(1)}, \\
-1, & \text { for } t \in l_{k}^{(2)},
\end{array} \quad h_{k}(t)=\left\{\begin{array}{ll}
1, & \text { for } t \in \tilde{l}_{k}^{(1)}, \\
-1, & \text { for } t \in \tilde{l}_{k}^{(2)}
\end{array} .\right.\right.
$$

Corollary. Theorem 2 implies the following equalities

$$
M_{k} P=\tilde{\delta}_{k} M_{k}, M_{k} Q=\left(1-\tilde{\delta}_{k}\right) M_{k}, P M_{k}=M_{k} \tilde{\delta}_{k} I, Q M_{k}=M_{k}\left(1-\tilde{\delta}_{k}\right) I,
$$

where $\delta_{k}=\frac{1+h_{k}}{2}$ and $\tilde{\delta}_{k}=\frac{1+\tilde{h}_{k}}{2}$.

In what follows, we will assume that the numbers $\alpha_{k}(k=1, \ldots, m)$ are such that $\Gamma_{j} \cap \Gamma \cap \tilde{\Gamma}_{k}=\varnothing(j, k=1, \ldots, m)$.

Theorem 4. Let $a \in L_{\infty}(\Gamma)$, then the operators $M_{j} a M_{k}(j, k=1, \ldots, m)$ are completely continuous in the space $L_{p}(\Gamma)$. If $a, b \in N_{k}$, then the following operators are also completely continuous:

$$
\mathrm{PaQbM}_{k}, Q a P b M_{k}, M_{k} a P b Q, M_{k} a Q b P, \operatorname{PaM}_{k} b Q, Q a M_{k} b P .
$$

Theorem 5 (main). Let $a, b \in \cap_{1 \leq k \leq m} N_{k}$ and $c_{k} \in L_{\infty}(\Gamma)$. In order for the operator

$$
A=a P_{\Gamma}+b Q_{\Gamma}+\sum_{k=1}^{m} c_{k} M_{k}
$$

to admit regularization in the space $L_{p}(\Gamma)$ it is necessary and sufficient that regularization was allowed by the operator

$$
A_{0}=a P+b Q .
$$

If the operator $A_{0}$ admits regularization, then

$$
\text { Ind } A=\text { Ind } A_{0} \text {. }
$$

The proof of this theorem uses the following lemma.

Lemma 1. The operator $H=I+\sum_{k=1}^{m} c_{k} M_{k}$ admits regularization in the space $L_{p}(\Gamma)$ and its index is zero.

\section{Example}

Let $\alpha_{1}=2$ and $\alpha_{2}=-2$. In this case $\tilde{\Gamma}_{1}=\Gamma_{2}, \tilde{\Gamma}_{2}=\Gamma_{1}, \Gamma_{1} \cap \Gamma \cap \tilde{\Gamma}_{2}=\{-1\}$ and

$$
\left(M_{1} \varphi\right)(t)=\frac{1}{\pi i} \int_{\Gamma} \frac{\varphi(\tau)}{\tau-t-2} d \tau,\left(M_{2} \varphi\right)(t)=\frac{1}{\pi i} \int_{\Gamma} \frac{\varphi(\tau)}{\tau-t+2} d \tau
$$

We denote by $K$ the operator $K=M_{1}+M_{2}$ and by $N$ - the set of piecewise continuous functions on $\Gamma$ and continuous at the points $\tau= \pm 1$.

Theorem 6. Let $a, b \in N$. For the operator $A=a I+b S+K$ to be Noetherian in the space $L_{p}(\Gamma)$, it is necessary and sufficient that the operator $A_{0}=a I+b S$ to have the same property. If operator $A_{0}$ is Noetherian, then $\operatorname{Ind} A=\operatorname{Ind} A_{0}$.

The proof of this theorem is based on a number of properties of the operator $K$, which we establish in the following lemmas. 
XX Міжнародний симпозіум «Методи дискретних особливостей в задачах математичної фізики/Discrete Singularities Methods in Mathematical Physics»,

\section{МДОЗМФ/DSMMPh-2021}

Lemma 2. For every function $h \in N$ there is a function $\tilde{h} \in N$ such that the operator $K h-\tilde{h} K$ is compact in $L_{p}(\Gamma)$. Moreover, if $h(t \pm 0) \neq 0(t \in \Gamma)$ then $\tilde{h}(t \pm 0) \neq 0$ too.

This statement is easily deduced from [5].

Lemma 3. The following relations are valid:

$$
S K=K, \quad K S=-K, \quad K^{2}=0 .
$$

Proof. Let $\varphi(t)=\sum_{k=-n}^{n} a_{k} t^{k}$ be a trigonometric polynomial, $\varphi_{+}(t)=\sum_{k=0}^{n} a_{k} t^{k}$ and $\varphi_{-}(t)=\sum_{k=-n}^{-1} a_{k} t^{k}$. Then $(S \varphi)(t)=\varphi_{+}(t)-\varphi_{-}(t)$ and for each point $t \in \Gamma \backslash\{-1,1\}$ the equality

$$
(K \varphi)(t)=-2 \sum_{k=-n}^{-1} a_{k}\left((t+2)^{k}+(t-2)^{k}\right) \text { is true. }
$$

It is easy to show that $S K \varphi=K \varphi$ and since the set of trigonometric polynomials is dense in the space $L_{p}(\Gamma)$, then $S K=K$. Further we have

$$
K S \varphi=K\left(\varphi_{+}-\varphi_{-}\right)=2 \sum_{k=-n}^{-1} a_{k}\left((t+2)^{k}+(t-2)^{k}\right)=-K \varphi .
$$

So $K S=-K$. The last relation from (13) easily follows from the first two lemmas. The lemma is proved.

Note also that the statement of Lemma 1 holds for operators of the form $F=I+f K$ $(f \in N)$.

Lemma 4. The operator $F=I+f K$ is Noetherian and $\operatorname{Ind} F=0$.

Proof of the theorem 6. If the operator $A_{0}=a I+b S$ is Noetherian in $L_{p}(\Gamma)$, then (see [5]) the conditions

$$
a(t \pm 0)+b(t \pm 0) \neq 0 \text { and } a(t \pm 0)-b(t \pm 0) \neq 0 \quad(t \in \Gamma) \text { are true. }
$$

Let $f$ denote the function $f=1 /(a+b)$. Based on Lemmas 2 and 3, we see that the operator $A$ can be represented in the form

$$
A=A_{0}(I+f K)+T,
$$

where $T$ is a compact operator. By Lemma 4, the operator $F=I+f K$ is Noetherian and Ind $F=0$. Therefore, operator $A$ is also Noetherian and $\operatorname{Ind} A=\operatorname{Ind} A_{0}$. The sufficiency has been proven.

Let us prove the necessity of the conditions of the theorem. Suppose that the operator $A=a I+b S+K$ is Noetherian, and the operator $A_{0}=a I+b S$ is not Noetherian. Let $\varepsilon$ be a positive number such that all operators $A^{\prime}$, satisfying the condition $\left\|A-A^{\prime}\right\|<\varepsilon$, are Noetherian and Ind $A^{\prime}=$ Ind $A$. Just as in [2], we can construct two Noetherian operators $B_{j}=a_{j} I+b_{j} S(j=1,2)$, such that $\left\|A_{0}-B_{j}\right\|<\varepsilon$ and Ind $B_{1} \neq$ Ind $B_{2}$. By virtue of what was proved above, the operators $A_{j}=a_{j} I+b_{j} S+K \quad(j=1,2)$ are Noetherian and Ind $A_{j}=$ Ind $B_{j}$. Therefore, Ind $A_{1} \neq \operatorname{Ind} A_{2}$. And since $\left\|A-A_{j}\right\|<\varepsilon \quad(j=1,2)$, then Ind $A_{1}=$ Ind $A_{2}$. The resulting contradiction proves that the operator $A_{0}$ is Noetherian. The theorem is proved. 


\title{
REFERENCES
}

1. Vornicescu G., Neagu V. Noetherian criteria for some perturbed integral operators. Studia Universitatis, Moldova State Universyty. 2020, pp. 17-25.

2. Krupnik N. $\Phi$-admissible perturbations of generalized operators of local type. Izv. Vuzov, 1985, №3, pp. 29-39.

3. Vasilevski N. On a class of singular integral operators with kernels of polarlogarithmic type. Izv. Akad. Nauk SSSR, Ser. Math. 1976, №1, pp. 133-151.

4. Neagu V. Essential spectrum of perturbed singular integral operators. ROMAI Journal, 2014, №1, pp. 105-121.

5. Krupnik N. Banach algebras with symbol and singular integral operators. Birkhäuse, Basel-Boston, 1987, 138 p.

6. Gohberg I., Krupnik N. Banach algebras generated by singular integral operators. Colloquia mathematica societatis Janos Bolyai 5. Hilbert space operators. 1970, Tihany (Hungary), pp.240-263.

7. Krasnoseliskii M. On a theorem of Riesz. Dokl. Akad. Nauk SSSR, 1960, 131, №2, pp. 246-248.

Надійшла 21.05.2021.

\section{Регуляризация некоторых возмущённых интегральных операторов в простраствах $L_{p}$}

\author{
Василе Нягу \\ Молдавский государственный университет, Кишинёв \\ E-mail: vasileneagu45@gmail.com
}

В статье представлены некоторые обобщения и уточнения статьи [1]: построены примеры интегральных (некомпактных) операторов с точечными особенностями, которые являются допустимыми возмущениями нётеровых операторов; установлена связь между регуляризаторами возмущенного и исходного операторов и доказано равенство индексов возмущенного и исходного операторов. Представленные результаты основаны на полученных в статье формулах композиции оператора с ядром Коши и операторами с точечными особенностями.

Ключевые слова: возмущённый сингулярный оператор, нётеровы условия. 